Supporting Information

\title{
Highly Efficient Multicomponent Gel Biopolymer Binder Enables Ultrafast Cycling and Applicability in Diverse Battery Formats
}

Ling Ding, Rita Leones*, Ahmad Omar*, Jing Guo, Qiongqiong Lu, Steffen Oswald, Kornelius Nielsch, Lars Giebeler, Daria Mikhailova*

L. Ding, Dr. R. Leones, Dr. A. Omar, Dr. J. Guo, Q. Lu, Dr. S. Oswald, Prof. Dr. K. Nielsch, Dr. L. Giebeler, Dr. D. Mikhailova

Leibniz Institute for Solid State and Materials Research (IFW) Dresden e.V., Helmholtzstr. 20, 01069 Dresden, Germany

Prof. Dr. K. Nielsch

Technische Universität Dresden, Institute of Materials Science,

Helmholtzstr. 7, 01069 Dresden, Germany 


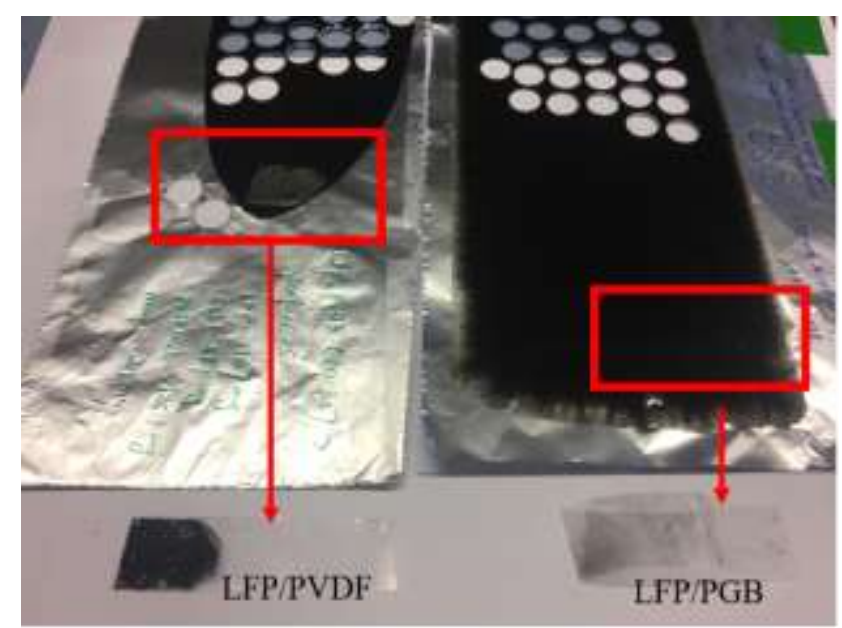

Figure S1. Simplified peeling test using tapes on LFP/PVDF and LFP/PGB electrodes. PGB: polymer gel binder. 

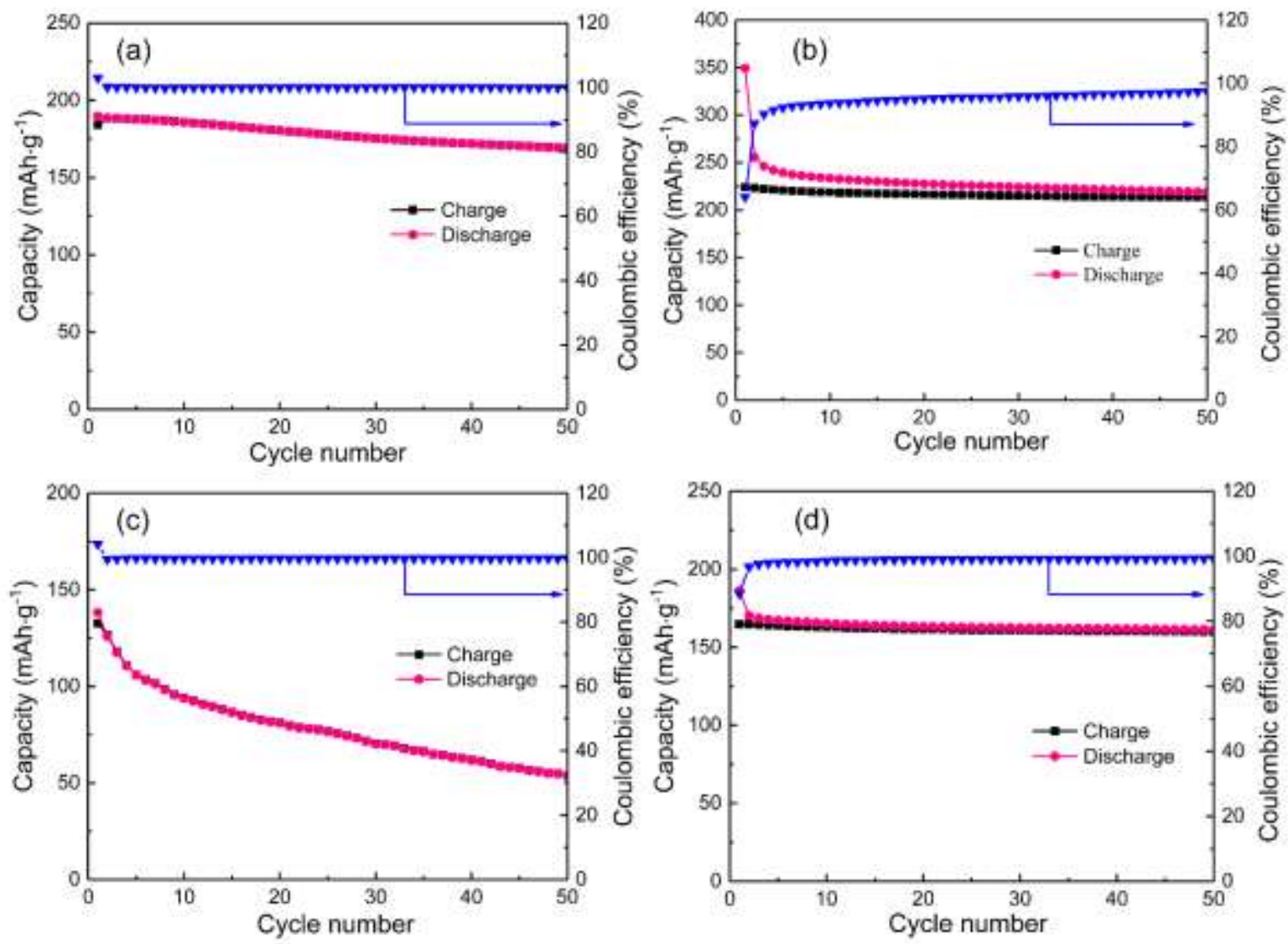

Figure S2. Cycling performance and Coulombic efficiency of (a) LFP/PGB (b) LTO/PGB (c) LFP/PVDF and (d) LTO/PVDF half cells cycled at low rate of $0.5 \mathrm{C}$. 


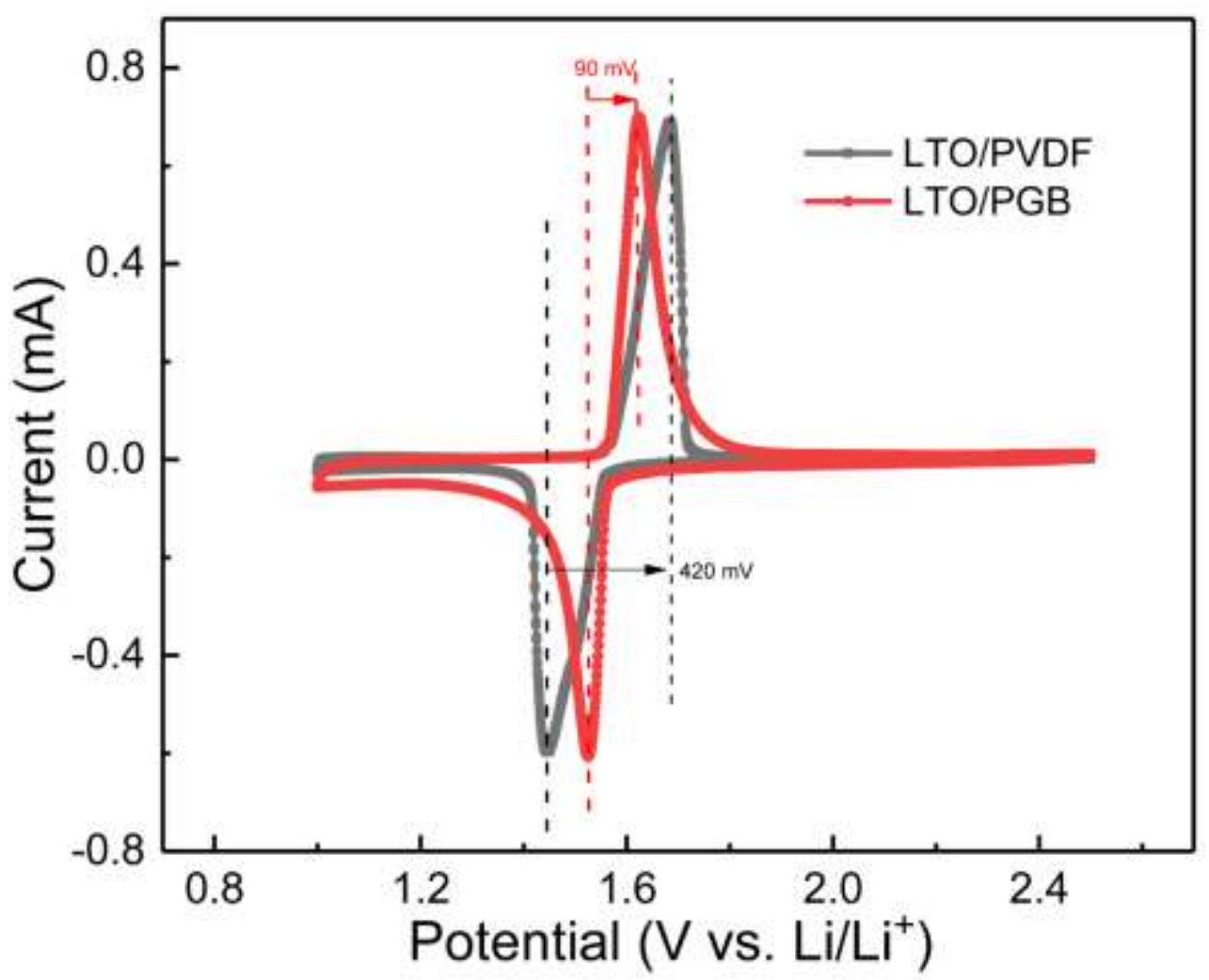

Figure S3. CV profiles of LTO/PGB and LTO/PVDF electrodes. The tests were performed in a voltage range of $1-2.5 \mathrm{~V}$ at a scanning rate of $0.1 \mathrm{mV} \cdot \mathrm{s}^{-1}$. 


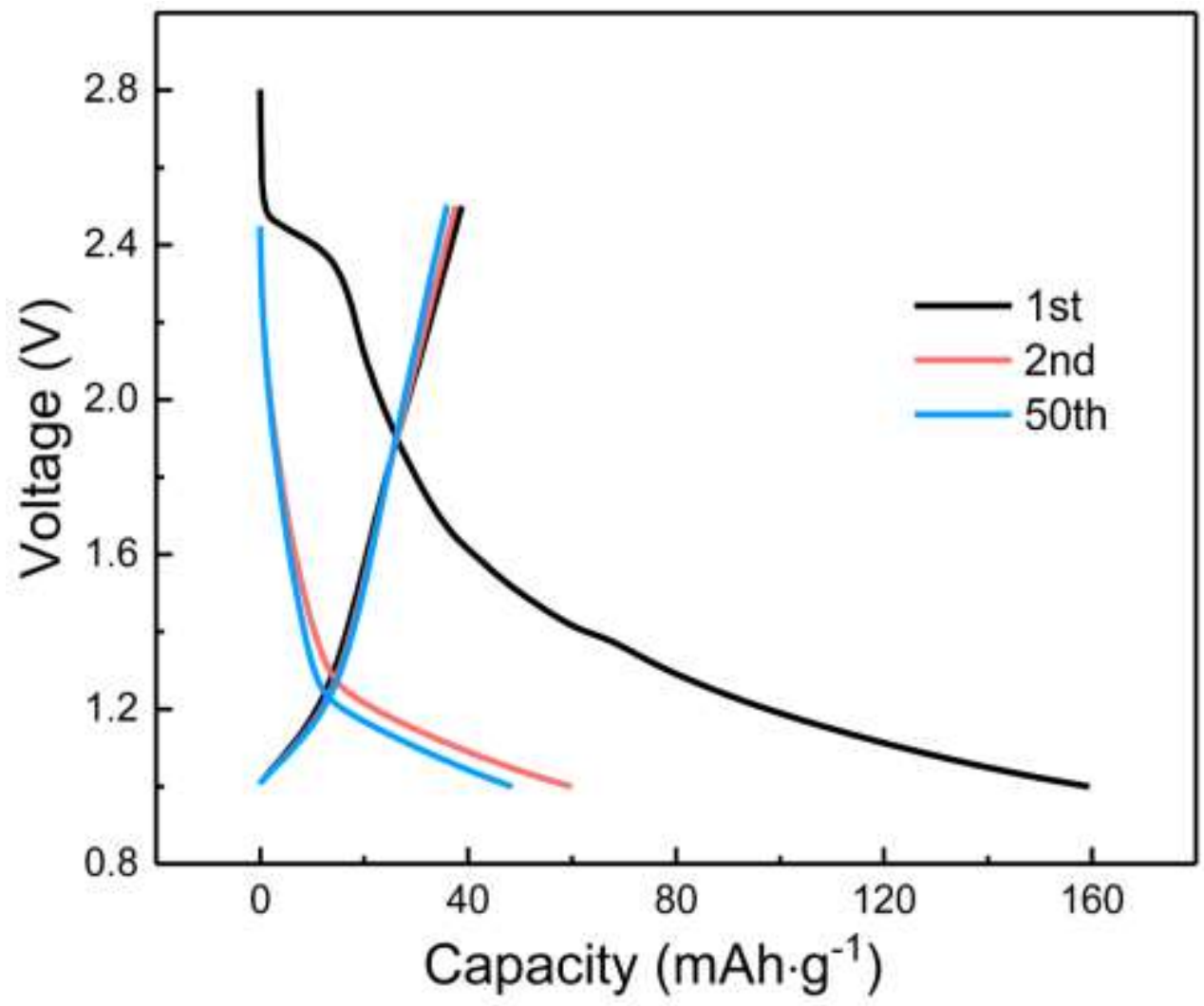

Figure S4. Charge/discharge voltage profiles of the electrode containing PGB and carbon black (prepared with a weight ratio of 1:1) cycled with a current density of 50 $\mathrm{mA} \cdot \mathrm{g}^{-1}$. 

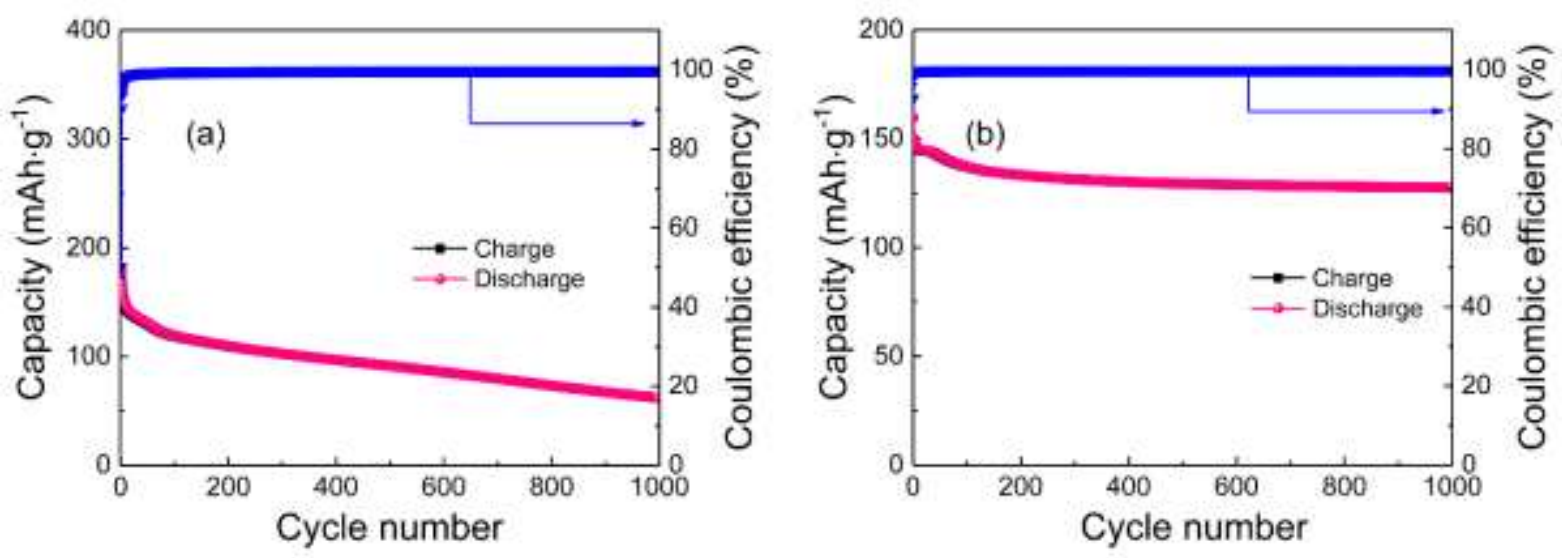

Figure S5. Cycling performance and Coulombic efficiency of (a) LTO/PGB and (b) LTO/PVDF half cells cycled at high rate of 10C. 


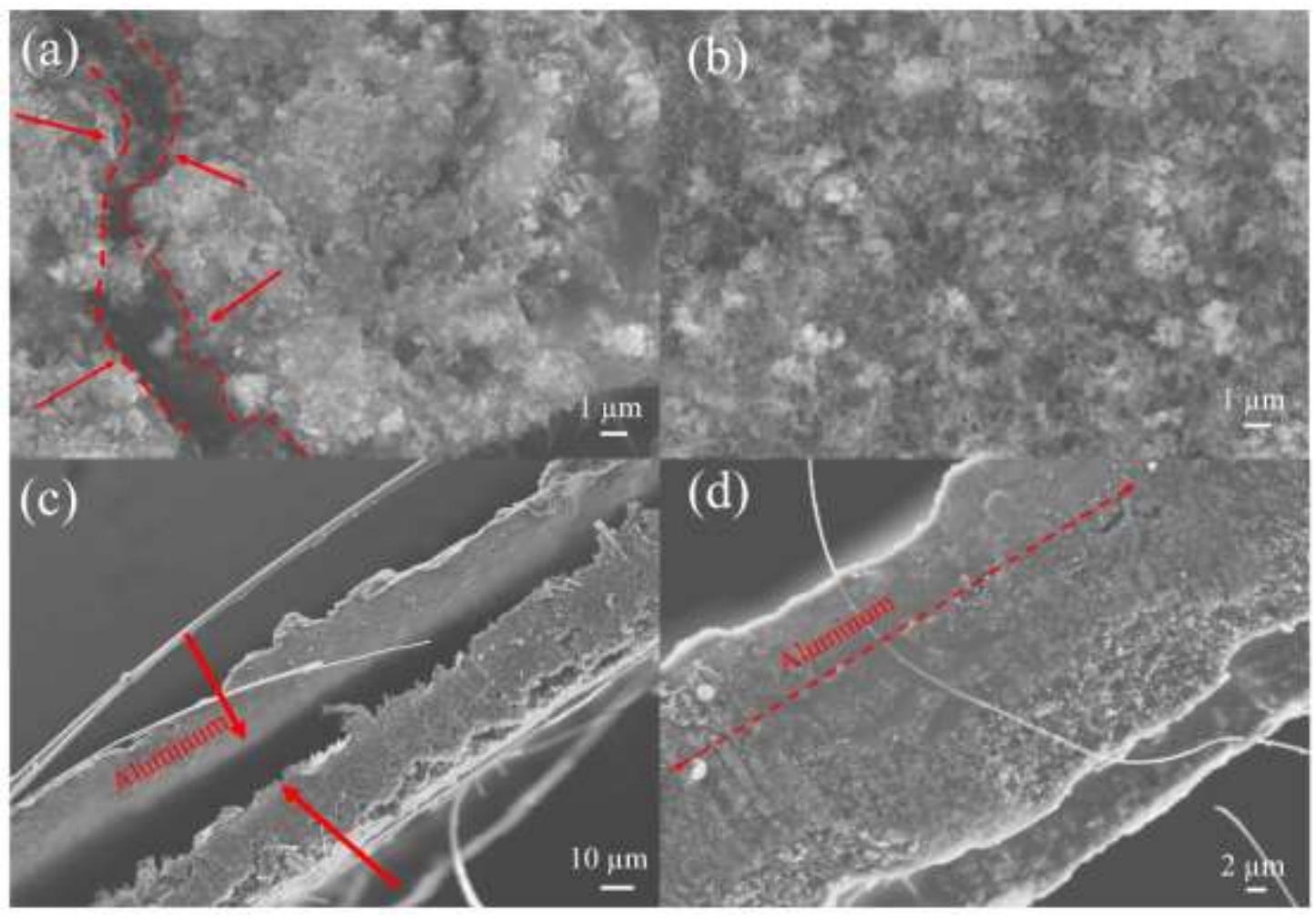

Figure S6. SEM images of (a) LFP/PVDF, (b) LFP/PGB surface after 200 cycles at 10C. Cross-sectional SEM images of (c) LFP/PVDF, (d) LFP/PGB after 200 cycles at $10 \mathrm{C}$. 

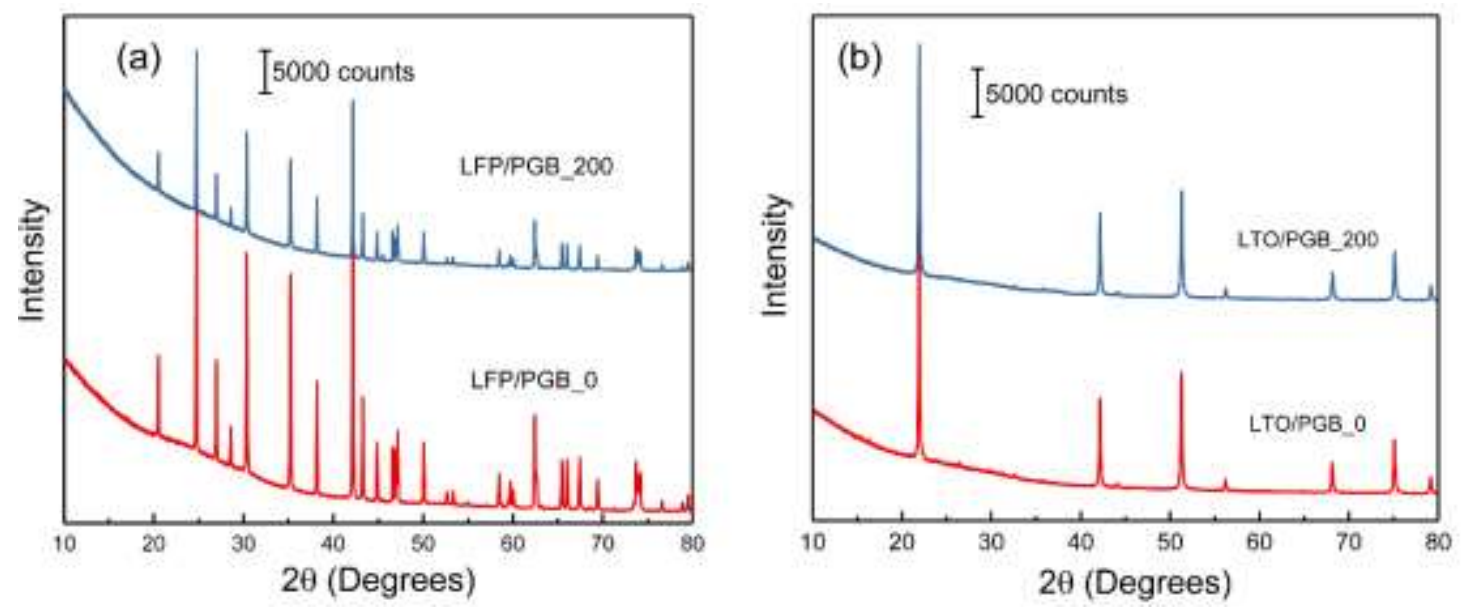

Figure S7. XRD patterns of electrodes before and after 200 cycles at 10C (a) LFP/PGB half cell (b) LTO/PGB half cell (performed using Co $\mathrm{K}_{\alpha 1}, \lambda=1.7889 \AA$ ). 


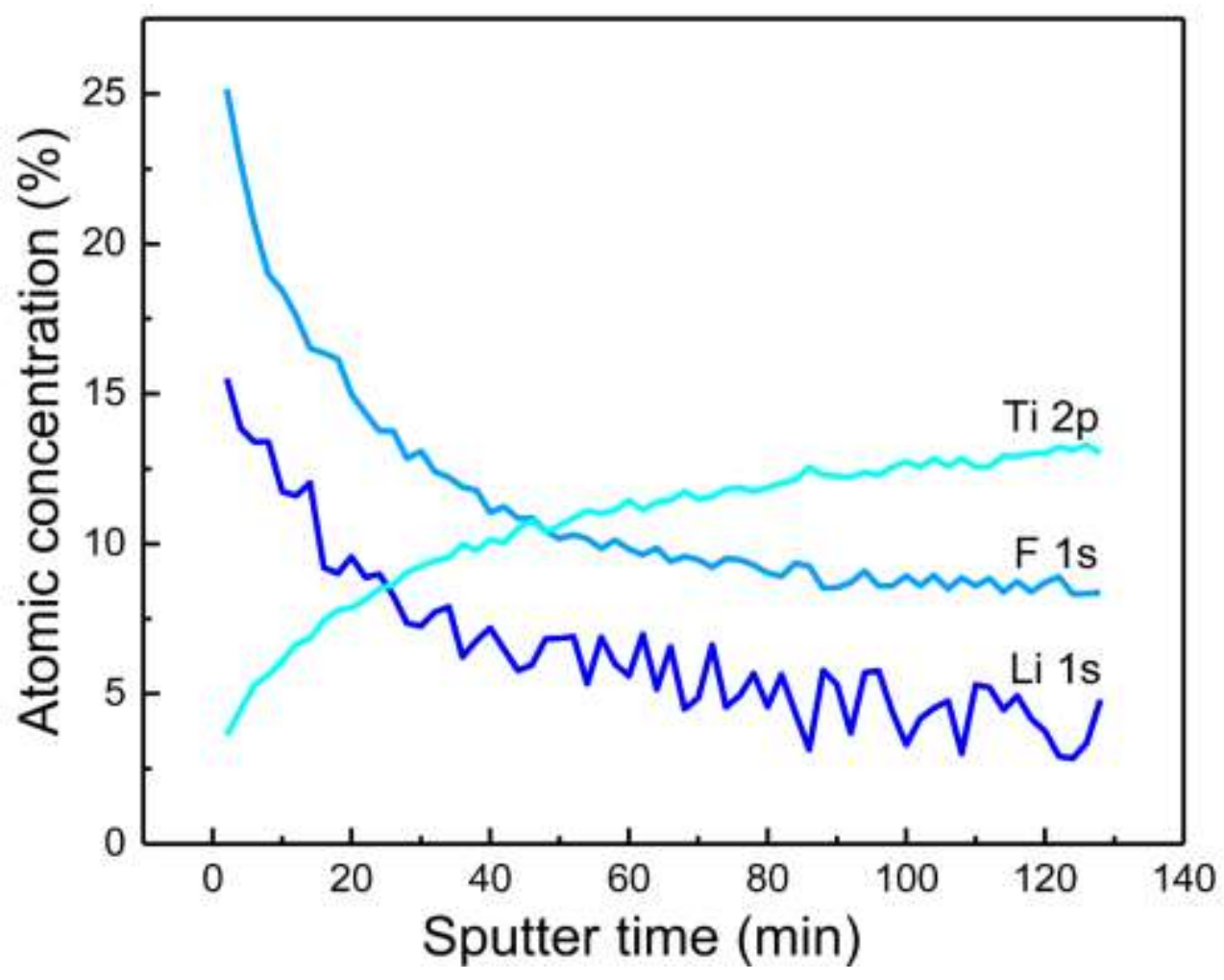

Figure S8. Depth profiling of a LTO/PGB electrode after 50 cycles at $10 \mathrm{C}$ vs. $\mathrm{Li} / \mathrm{Li}^{+}$. 

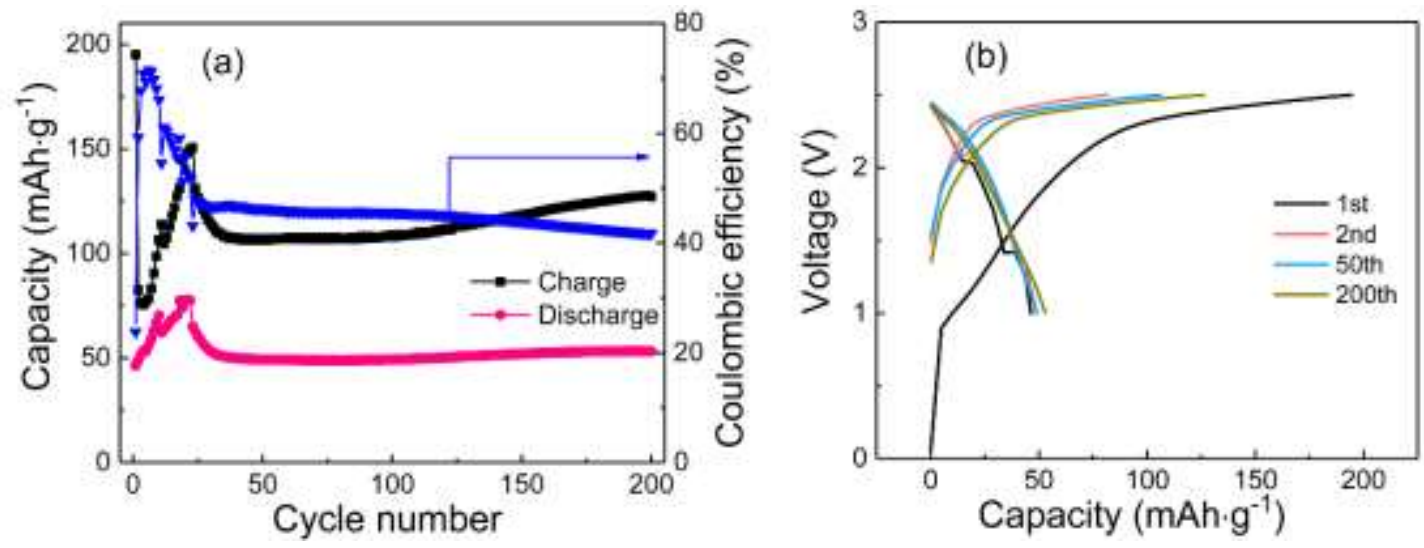

Figure 9. (a) Cycling performance and (b) charge/discharge voltage profiles of LFP\|LTO-PVDF full cell at 10C. 

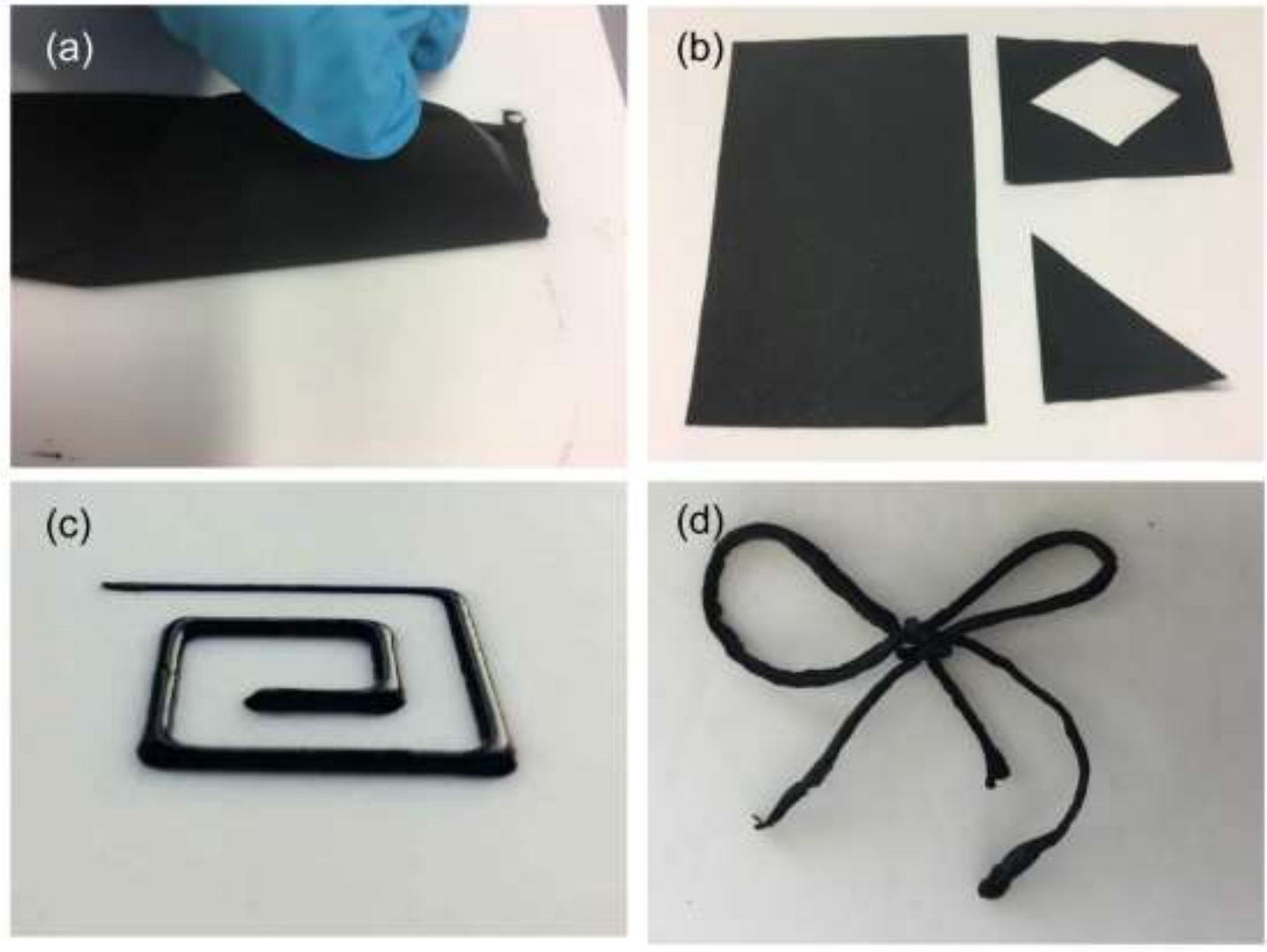

Figure S10. (a) The freestanding electrodes can be easily peeled off from the Teflon substrate and (b) can be cut into designed structures; (c) The electrode slurry can be directly printed into intricate structures as well as (d) extruded into flexible fibers which can be made into various shapes easily. 
Table S1. Comparison of electrochemical performance between LFP/PGB and LTO/PGB with previously reported literature utilizing different modifications of LFP and LTO.

\begin{tabular}{|c|c|c|c|}
\hline Modifications & $\begin{array}{l}\text { Capacity } \\
\left(\mathrm{mAh} \cdot \mathrm{g}^{-1}\right)\end{array}$ & Rate & $\begin{array}{c}\text { Particle } \\
\text { size }\end{array}$ \\
\hline LFP/PAB-C ${ }^{1}$ & $137.5\left(300^{\text {th }}\right)$ & $10 \mathrm{C}$ & \\
\hline C-LFP ${ }^{2}$ & $139.8\left(50^{\text {th }}\right)$ & $1 \mathrm{C}$ & $\sim 90 \mathrm{~nm}$ \\
\hline Nano-LFP ${ }^{3}$ & $123\left(1^{\mathrm{st}}\right)$ & $10 \mathrm{C}$ & $<65 \mathrm{~nm}$ \\
\hline C-LFP ${ }^{4}$ & $130\left(1^{\mathrm{st}}\right)$ & $5 \mathrm{C}$ & $146 \mathrm{~nm}$ \\
\hline LFP Nanoplates 5 & $148\left(1^{\mathrm{st}}\right)$ & $10 \mathrm{C}$ & $<200 \mathrm{~nm}$ \\
\hline LFP@G $\mathrm{G}^{6}$ & $139.8\left(600^{\text {th }}\right)$ & $10 \mathrm{C}$ & $<200 \mathrm{~nm}$ \\
\hline$L F P / P G B$ (this work) & $110\left(200^{\text {th }}\right)$ & $10 C$ & $\sim 400 \mathrm{~nm}$ \\
\hline N-LTO@TiC/C ${ }^{7}$ & $143\left(1^{\mathrm{st}}\right)$ & $10 \mathrm{C}$ & \\
\hline LTO-RTO/C ${ }^{8}$ & $121.6\left(200^{\text {th }}\right)$ & $2 \mathrm{C}$ & \\
\hline $\mathrm{LTO}^{-\mathrm{TiO}_{2}}{ }^{9}$ & $94.2\left(1^{\mathrm{st}}\right)$ & $15 \mathrm{C}$ & \\
\hline Hollow LTO microspheres ${ }^{10}$ & $139\left(1^{\mathrm{st}}\right)$ & $10 \mathrm{C}$ & \\
\hline Microporous LTO microbars ${ }^{11}$ & $141\left(1^{\mathrm{st}}\right)$ & $10 \mathrm{C}$ & \\
\hline$L T O / P G B$ (this work) & $112\left(200^{t h}\right)$ & $10 C$ & \\
\hline
\end{tabular}


Table S2. Elemental concentration measured by XPS on three LTO/PGB electrodes prepared under different conditions.

\begin{tabular}{lccccccccc}
\hline & Li 1s & C 1s & N 1s & O 1s & F 1s & P 2p & S 2p & Ti 2p & Cu 2p \\
\hline Pristine & 0.66 & 61.96 & 5.59 & 25.52 & 1.90 & 0.18 & 0.11 & 2.06 & 2.02 \\
Soaked & 1.34 & 54.68 & 6.71 & 21.73 & 9.84 & 0.77 & 0.23 & 2.47 & 2.26 \\
After 50 cycles & 11.63 & 37.94 & 1.05 & 14.49 & 30.22 & 3.89 & 0.48 & 0.13 & 0.18 \\
\hline
\end{tabular}




\section{References}

(1) Mo, Y.; Liu, J.; Meng, C.; Xiao, M.; Ren, S.; Sun, L.; Wang, S.; Meng, Y., Stable and Ultrafast Lithium Storage for $\mathrm{LiFePO}_{4} / \mathrm{C}$ Nanocomposites Enabled by Instantaneously Carbonized Acetylenic Carbon-Rich Polymer. Carbon 2019, 147, 19-26.

(2) Kim, H.J.; Bae, G.H.; Lee, S.M.; Ahn, J.-H.; Kim, J.K., Properties of Lithium Iron Phosphate Prepared by Biomass-Derived Carbon Coating for Flexible Lithium Ion Batteries. Electrochim. Acta 2019, 300, 18-25.

(3) Choi, D.; Kumta, P. N., Surfactant Based Sol-Gel Approach to Nanostructured $\mathrm{LiFePO}_{4}$ for High Rate Li-ion Batteries. J. Power Sources 2007, 163, 1064-1069.

(4) Konarova, M.; Taniguchi, I., Synthesis of Carbon-Coated $\mathrm{LiFePO}_{4}$ Nanoparticles with High Rate Performance in Lithium Secondary Batteries. J. Power Sources 2010, 195, 3661-3667.

(5) Wang, L.; He, X.; Sun, W.; Wang, J.; Li, Y.; Fan, S., Crystal Orientation Tuning of $\mathrm{LiFePO}_{4}$ Nanoplates for High Rate Lithium Battery Cathode Materials. Nano Lett. 2012, 12, 5632-5636.

(6) Yi, X.; Zhang, F.; Zhang, B.; Yu, W.J.; Dai, Q.; Hu, S.; He, W.; Tong, H.; Zheng, J.; Liao, J., (010) Facets Dominated $\mathrm{LiFePO}_{4}$ Nano-Flakes Confined in 3D Porous Graphene Network as A HighPerformance Li-Ion Battery Cathode. Ceram. Int. 2018, 44, 18181-18188.

(7) Yao, Z.; Xia, X.; Xie, D.; Wang, Y.; Zhou, C. a.; Liu, S.; Deng, S.; Wang, X.; Tu, J., Enhancing Ultrafast Lithium Ion Storage of $\mathrm{Li}_{4} \mathrm{Ti}_{5} \mathrm{O}_{12}$ by Tailored TiC/C Core/Shell Skeleton Plus Nitrogen Doping Adv. Funct.Mater. 2018, 28, 1802756.

(8) Wang, P.; Zhang, G.; Cheng, J.; You, Y.; Li, Y.K.; Ding, C.; Gu, J.-J.; Zheng, X.S.; Zhang, C.F.; Cao, F.-F., Facile Synthesis of Carbon-Coated Spinel $\mathrm{Li}_{4} \mathrm{Ti}_{5} \mathrm{O}_{12} / \mathrm{Rutile}-\mathrm{TiO}_{2}$ Composites as An Improved Anode Material in Full Lithium-Ion Batteries with $\mathrm{LiFePO}_{4} @$ N-doped Carbon Cathode. ACS Appl. Mater. Interfaces 2017, 9, 6138-6143.

(9) Gao, L.; Wang, L.; Dai, S.; Cao, M.; Zhong, Z.; Shen, Y.; Wang, M., $\mathrm{Li}_{4} \mathrm{Ti}_{5} \mathrm{O}_{12}-\mathrm{TiO}_{2}$ Nanowire Arrays Constructed with Stacked Nanocrystals for High-Rate Lithium and Sodium Ion Batteries. J. Power Sources 2017, 344, 223-232.

(10) Zhu, K.; Gao, H.; Hu, G.; Liu, M.; Wang, H. Scalable Synthesis of Hierarchical Hollow $\mathrm{Li}_{4} \mathrm{Ti}_{5} \mathrm{O}_{12}$ Microspheres Assembled by Zigzag-Like Nanosheets for High Rate Lithium-Ion Batteries. J. Power Sources 2017, 340, 263-272.

(11) Tang, L.; He, Y. B.; Wang, C.; Wang, S.; Wagemaker, M.; Li, B.; Yang, Q. H.; Kang, F. High-Density Microporous $\mathrm{Li}_{4} \mathrm{Ti}_{5} \mathrm{O}_{12}$ Microbars with Superior Rate Performance for Lithium-Ion Batteries Adv. Sci. 2017, 4, 1600311. 